This is the accepted version of the article:

Guardingo, M.; Bellido, E.; Miralles-Llumà, R.; Faraudo, J.; Sedõ, J.; Tatay, S.; Verdaguer, A.; Busqué, F.; Ruiz-Molina, D.. Bioinspired catechol-terminated self-assembled monolayers with enhanced adhesion properties. Small, (2014). 10. 8: 1594 - . 10.1002/smll.201302406.

Available at: https://dx.doi.org/10.1002/smll.201302406 


\title{
Bioinspired catechol-terminated self-assembled monolayers with enhanced adhesion properties
}

\author{
By Mireia Guardingo, Elena Bellido, Rosa Miralles-Llumà, Jordi Faraudo, Josep Sedó,
} Sergio Tatay, Albert Verdaguer, Felix Busqué and Daniel Ruiz-Molina*

[*]M. Guardingo, Dr. E. Bellido, Dr. A. Verdaguer, Dr. J. Sedó, Dr. D. Ruiz-Molina ICN2 - Institut Catala de Nanociencia i Nanotecnologia, Campus UAB, 08193 Bellaterra (Barcelona), Spain CSIC

CSIC - Consejo Superior de Investigaciones Cientificas, ICN2 Building, Campus

UAB ,08193 Bellaterra (Barcelona), Spain

E-mail: druiz@cin2.es

R. Miralles-Llumà, Dr. F. Busqué

Departament de Química, Universitat Autònoma de Barcelona

Campus UAB- 08193

Cerdanyola del Vallès (Barcelona)-Spain

Dr. J. Faraudo

Insitut de Ciència de Materials de Barcelona (CSIC)

Campus UAB, 08193,

Cerdanyola del Vallès (Barcelona)-Spain

Dr. S. Tatay

Instituto de Ciencia Molecular (ICMol)

Universidad de Valencia, Catedrático José Beltrán 2, 46890 Paterna (Valencia)-Spain

Keywords: catechol, biomimetic, adhesive surface, SAMs, polydopamine

\section{Introduction}

Polydopamine (PDA) has emerged in recent years as a highly versatile bio-inspired coating and adhesive primer, after the pioneering research by Messersmith and co-workers. ${ }^{[1]}$ These authors described an easy and straightforward coating method based on the aerobic autooxidation of dopamine in mildly basic aqueous media, and simultaneous in situ deposition of the resulting polymer. ${ }^{[2]}$ Such methodology was shown to be effective on a wide variety of substrates, ranging from inorganic (e.g. metals, metal oxides) to organic (e.g. polymers), including notoriously difficult materials, such as PTFE, ${ }^{[3]}$ with a remarkably wide-ranging effectiveness. ${ }^{[4]}$ Since this seminal work, the possibilities of polydopamine as primer coating have been thoroughly explored and used in cell adhesion, ${ }^{[5]}$ nanoparticle coating and 
assembly, ${ }^{[6,7]}$ drug delivery, ${ }^{[8]}$ and membrane modification ${ }^{[9]}$ among others. Moreover, alternative synthetic approaches to obtain polydopamine-like materials with similar coating efficiencies have also been reported. ${ }^{[10]}$

Such adhesion properties are mostly attributed to the catechol moieties present in the PDA structure. Notwithstanding its usefulness, the structure of PDA is still subject of debate, being consistent with an association of chemically disordered oligo-/polymeric scaffolds containing both catecholic and $o$-quinoid moieties. ${ }^{[11]}$ Its structural disorder, together with the typical irregular topography of polymeric coatings, makes it very difficult to gain details on the PDA surface characteristics, which are generally considered to be determined by the outermost 5$10 \AA$ A layer. ${ }^{[12]}$ In this context, catechol-based self-assembled monolayers (SAMs) arise as a promising research alternative to establish meaningful structure-property relationships.

SAMs are molecular assemblies of organic adsorbates that spontaneously organize on surfaces in an orderly fashion by an easy adsorption process. ${ }^{[13]}$ Head groups self-assemble together on suitable surfaces forming domains of close-packed molecules where the tail (outward facing) groups determine the characteristics of the coated surface. Previous examples of catechol-terminated SAMs have been reported so far, ${ }^{[14]}$ though most of the work focuses on their electrochemical behaviour. ${ }^{[15]}$ As far as we know, the adhesive properties of catechol-terminated monolayers are yet to be quantified and studied in detail. Herein we report the study of SAMs of 4-(6'-mercaptohexyl)catechol (1) on different gold surfaces by both experimental and theoretical means. The adhesive properties of the resulting SAMs are studied at the local scale by means of Atomic Force Microscopy (AFM), and compared with those of ODT- and polydopamine-coated substrates. Results show some of the main factors explaining the enhanced adhesion properties of SAMs of compound $\mathbf{1}$ as well as the influence that the catechol group has on the structure and formation of the monolayer. 


\section{Results}

\subsection{Synthesis and self-assembly of Compound 1}

The synthesis of target thiol 1 was achieved through a convergent synthetic approach, as shown in Scheme 2. The aldehyde moiety of commercially available 3,4dibenzyloxybenzaldehyde was reduced with $\mathrm{NaBH}_{4}$ to give the corresponding alcohol, which was then treated with $\mathrm{PBr}_{3}$ to afford the bromoderivative 2 in $89 \%$ overall yield. Afterwards, compound 2 was quantitatively transformed in the corresponding phosphonium bromide salt following a reported procedure. ${ }^{[16]}$ On the other hand, the already described 5-bromopentanal was obtained from 5-bromobutanol in $87 \%$ yield by oxidation with PCC. ${ }^{[17]}$ Combination of the two fragments in a Wittig reaction, using $\mathrm{K}_{2} \mathrm{CO}_{3}$ as a base, gave a 1:1 mixture of olefins $Z$ - and $E$ - 4 in $50 \%$ yield. The use of stronger bases such as $n$-BuLi proved to be less efficient for this transformation. Next, hydrogenation of the mixture of olefins $\mathbf{4}$ under palladium catalysis resulted in simultaneous deprotection of the alcohol groups and reduction of the double bond, affording the corresponding $\omega$-bromocatechol in $86 \%$ yield. After treatment with KSAc, the thioacetate was obtained in $85 \%$ yield. Finally, hydrolysis of the thioacetate group in basic conditions afforded a mixture of the target thiol $\mathbf{1}$ (49\% yield) and its corresponding disulfide (13\% yield), which were separated by column chromatography (For experimental details and characterization of intermediates see Supporting Information, section $5)$.

\section{-Insert Scheme 2 here-}

SAMs of catechol 1 were obtained following standard protocols on two different substrates; namely, epitaxial gold on mica (RMS $\sim 0.3 \mathrm{~nm}$ ), and polycrystalline gold on $\mathrm{Si} / \mathrm{SiO}_{2}(\mathrm{RMS} \sim 1$ nm) (see Experimental Section). In a typical experiment, a clean substrate was immersed in a $1 \mathrm{mM}$ ethanolic solution of $\mathbf{1}$ for 18 hours at ambient conditions and afterwards thoroughly 
washed with fresh ethanol (see AFM images in the Supporting Information, Figure S3). The resulting catechol-terminated SAMs were characterized using Polarization ModulationInfrared Reflection-Absorption Spectroscopy (PM-IRRAS), X-Ray Photoelectron Spectroscopy (XPS) and Spectroscopic Ellipsometry. The PM-IRRAS spectrum (see Supporting Information, Figure S1 and Table S1) is in agreement with those previously reported for related catechol-terminated SAMs. ${ }^{[18,14 c-d, 13 a]}$ Vibrational bands ranging from 2960 to $2855 \mathrm{~cm}^{-1}$, which are also present in the corresponding ATR spectrum of a bulk sample, are assigned to the stretching modes of $\mathrm{C}-\mathrm{H}$ bonds in the alkyl chain. Importantly, the band around $2495 \mathrm{~cm}^{-1}$ assigned to the $\mathrm{S}-\mathrm{H}$ bond stretching is not observed in the monolayer spectra, fully consistent with thiol groups covalently bound to the Au substrate. The presence of the hydroxyl groups is confirmed by the peaks at $3462 \mathrm{~cm}^{-1}, 1264$ and $1113 \mathrm{~cm}^{-1}$, assigned to $\mathrm{O}-\mathrm{H}$ stretching and in-plane bending, and C-O stretching modes. Peaks at 1522 and 1458 $\mathrm{cm}^{-1}$ are assigned to the in-plane stretching of the $\mathrm{C}=\mathrm{C}$ bonds of the aromatic ring. ${ }^{[19]}$ Overall, the high intensity of the signals associated to the $\mathrm{C}-\mathrm{O}, \mathrm{O}-\mathrm{H}$, and $\mathrm{C}=\mathrm{C}$ bonds discard the possibility of a parallel orientation of the aromatic ring with regard to the surface -which would be expected if the adsorbates were lying flat on the substrate-, and hence suggest that the thiols are standing in an orderly fashion with a certain tilt angle. ${ }^{[20]}$ Finally, a very weak signal appears at $1666 \mathrm{~cm}^{-1}$, indicating the presence of traces of oxidized (o-quinoid) catechol species.

XPS results are also in agreement with those found for similar catechol-terminated monolayers ${ }^{[14 d, 17]}$ (See Supporting Information, Figure S2). The S 2p core level clearly presents two peaks with a 2:1 ratio, located at 161.9 and $163.0 \mathrm{eV}$, assigned to sulphur atoms chemically bound to gold surfaces. A more detailed deconvolution of this spectral region suggests that a small amount (less than 10\%) of unbound sulphur species may also be present in the sample. Most likely, these signals arise from disulfides that spontaneously generate when the thiol is in solution, as observed during the synthesis of $\mathbf{1}$. The XPS C 1s core level is 
deconvoluted into three peaks at $\sim 284.3,284.5$ and $286.0 \mathrm{eV}$ (peak ratio 2:3:1), corresponding to four aromatic H-bound carbons, six aliphatic, and two O-bound aromatic carbons, respectively. Although oxidation of the catechol moiety to $o$-quinone is feasible in an oxygen atmosphere, no significant contribution from this moiety is observed by XPS, in accordance with PM-IRRAS spectra. Importantly, the ratio of intensities between the C 1s and the S 2p signals measured on the same sample at different take off-angles (TOA) increases when the measurement is performed at $\mathrm{TOA}=60^{\circ}$. This indicates that $\mathrm{S}$ atoms are preferentially located close to the surface, while $\mathrm{C}$ atoms tend to position away from it, in agreement with what should be expected for a S-bound monolayer. ${ }^{[21]}$

Finally, the estimation of film thickness was performed using spectroscopic Ellipsometry in the range between $300-400 \mathrm{~nm}$. According to its UV-Vis spectra, 1 does not absorb light in this wavelength range, so we chose to model the film with a transparent medium with a refractive index of $1.49 .{ }^{[22,23]}$ Film thickness was found to be approximately $5 \AA$, being slightly dependent on the point of sampling. Since this value is well below the full-stretched length of the molecule -ca. $12 \AA-$, the formation of stacked multilayers was ruled out. Using this same molecular length value, an average tilt angle with respect to the surface of ca. 27 degrees may be calculated.

\subsection{Adhesive Properties and Surface Effects}

The adhesive properties of the catechol-terminated SAMs on epitaxial gold were studied by AFM force-distance (F-d) curves, a technique that had already been used to study the adhesive properties of dopamine. ${ }^{[24]}$ In a typical experiment, a non-functionalized AFM tip is brought into contact with a surface at a constant speed and then pressed against it to a fixed load; afterwards, the tip is retracted from the surface. During the whole process, the deflection of the cantilever is registered and plotted as a function of the extension of the piezoelectric 
sensor. Depending on the information to be derived from the experiments, different parts of the F-d curves should be analyzed. ${ }^{[25]}$ In our case, adhesion force values between the tip and the sample were calculated from the jump-out of the tip during the retraction movement (see Supporting Information, Fig. S3 and the Experimental Section for more details). Representative histograms of adhesion force were constructed from repeated force-distance curves registered across the surface. Two additional substrates -bare epitaxial gold and gold modified with an ODT monolayer-, were also studied for comparison purposes. The experiments were performed on the same experimental session, in order to minimize temperature and humidity fluctuations.

Bare gold substrates presented a force histogram centred at small values of 8-9 $\mathrm{nN}$ (see Figure 1a-b), most likely arising from capillary forces originated by the presence of a thin layer of water on top of the bare Au surface at ambient conditions. ${ }^{[26]}$ ODT-modified substrates showed adhesion force values around 5-6 nN (see Figure 1c-d). A possible explanation for such small values would lie in the higher hydrophobicity of the ODT coating, on top of which the water layer is expected to be considerably thinner, resulting in weaker adhesion to the AFM tip. It was also observed that the F-d curves obtained for the ODT monolayer presented a different shape for the retraction movement, with a softer and less vertical jump-out, in contrast with bare Au substrates. This can be attributed to deformation of the monolayer caused by the tip, as well as to irregularities of the thin top water layer. Finally, measurements on catechol 1-modified surfaces afforded a set of F-d values centred at $45 \mathrm{nN}$ (see Figures 1e-f). Overall, the catechol-terminated monolayer showed an average adhesion force at the nanoscale level five times higher than that measured for bare gold, and eight times higher than that of an ODT monolayer.

\section{-Insert Figure 1 here-}


For comparison purposes, additional F-d measurements were recorded on a polydopamine coating obtained following the procedure already described in the literature ${ }^{[1]}$ Epitaxial gold substrates were kept in vertical orientation while immersed in a stirred aqueous solution of dopamine hydrochloride ( $\mathrm{pH}$ 8.5) for an hour and then rinsed with Milli-Q water (see Experimental Section for more details). AFM imaging of PDA-coated substrates revealed a rough topography formed by small aggregates deposited on the surface, in agreement with previously reported data ${ }^{[27]}$ (see Supporting Information Figure S4). Afterwards, F-d curves were recorded and represented in the histogram shown in Figure 2. In some experiments, PDA coatings showed multiple jump-off or deformed curves with a high degree of statistical dispersion, associated to plastic deformation of the coating under the pressure of the tip. Comparison with values obtained for catechol 1-terminated SAMs shows that a) the average adhesion of the catechol monolayer is slightly higher than the maximum adhesion force recorded on the PDA thin film, and b) much more consistent results are obtained on SAMs owing to their intrinsically homogenous nature (both chemical and spatial).

\section{-Insert Figure 2 here-}

Further studies were carried out to assess the effect of surface roughness on monolayer properties. For this, SAMs of 1 were prepared on polycrystalline gold substrates exhibiting rough topographies (RMS 1 nm), (see Figure S4 Supplementary Material), as opposed to the atomically flat substrates studied in the previous case. F-d curves recorded on substrates coated after long immersion times $(18 \mathrm{~h})$ afforded a high dispersion of values, and no better adhesion was observed with respect to a pristine gold substrate. This observation is attributed to an increase in the distance between neighbouring catechol tail groups as surface roughness increases, ${ }^{[28]}$ leading to the deposition of a poorly packed monolayer, and consequently a decrease in the adhesion force. Moreover, the rougher topography of the substrate is expected 
to lead to important variations in the contact geometry between the tip and the sample, thus adding uncertainty to the results. As an alternative to AFM measurements, the same coated substrate was immersed in a colloidal solution of iron oxide nanoparticles $(\varnothing \sim 8-10 \mathrm{~nm})$ and sonicated for 15 min. ${ }^{[13 a]}$ A relatively homogenous distribution of nanoparticles was observed across the whole surface, as shown in Figure 3. This procedure was repeated for comparison purposes on two additional polycrystalline gold surfaces; namely, an unmodified, bare substrate and a polydopamine-coated substrate. Very few nanoparticles were found randomly adsorbed on bare gold substrates, while the same experiment on the polydopamine-coated samples was not conclusive due to damage of the coating upon sonication (see Supporting Information, Figure S5). Therefore, despite the fact that roughness of the substrate impedes a precise assessment of the monolayer adhesion by AFM, the thiols seem to be still homogeneously distributed on catechol-coated surfaces, with enough catechol groups available for NP attachment.

\section{-Insert Figure 3 here-}

\subsection{Monolayer Formation Process}

The alkanethiol adsorption and equilibration time needed for the formations of SAMs has been thoroughly studied and is assumed to occur in a two-step mechanism influenced by the chemical nature of the head groups. ${ }^{[29]}$ However, the vast majority of these studies are based on long chain alkanethiols, whose chemical nature is only roughly comparable to compound $\mathbf{1}$. In our case, the importance of equilibration time on the adhesive properties of the monolayer was studied by immersing a clean epitaxial gold substrate in a $1 \mathrm{mM}$ ethanolic solution of $\mathbf{1}$ at ambient conditions for only 15 minutes. F-d curves recorded following the experimental procedure described above (See section 2.2) showed adhesion force values centred around 5-6 
$\mathrm{nN}$ (see Supplementary Material, Figure S5), far below those found for catechol-terminated SAMs with longer immersion times, and otherwise rather similar to those obtained for long alkyl chains. Following a previous approximation, a polycrystalline gold substrate that had been kept in the thiol solution for a short period of time (15 min) was then immersed in a dispersion of iron oxide nanoparticles. The particle coverage obtained on this substrate was shown to be poor and inhomogeneous. Overall, these results would suggest that transient layers (i.e. before equilibrium) afford surfaces with essentially non-adhesive character. Confirmation for the need of having sufficiently prolonged immersion times to obtain good quality monolayers was obtained by fabricating monolayer dot arrays with a direct-write scanning probe lithography technique such as Dip-Pen Nanolithography (DPN). ${ }^{[30]}$ This methodology has been previously shown to form close-packed and highly ordered SAMs with commonly used thiols such as ODT and mercaptohexadecanoic acid provided that appropriate deposition and solvent evaporation conditions are chosen. ${ }^{[31]}$ Contact mode AFM images obtained immediately after the deposition using the same coated tip showed a difference in friction on the spots where the dip pen deposition procedure was performed, proving that the catechol-terminated thiol had efficiently transferred to the surface (Figure 4a).

Arrays of deposited catechol $\mathbf{1}$ were then located by LFM imaging using a clean tip (Figure 4b). F-d curves were recorded afterwards on both functionalised and pristine areas while scanning the surface with the same probe. No significant differences were observed between the adhesion values of bare gold and the coated areas, showing that droplet evaporation takes places before the equilibrium conditions for the SAM formation are achieved. These results were reproducible for additional square motifs obtained with slow writing speeds and repeated passes, which should have contributed to improve the monolayer quality. It is important to point out here that at least one preliminary scan of the area is required to locate the arrays before measuring the adhesion, meaning that both the surface and the tip can be modified in this process thus affecting the subsequent measurements. ${ }^{[32]}$ Nevertheless, in view 
of these results we can conclude that no significant enhanced adhesion could be measured on sub-monolayers prepared by DPN, suggesting that this technique affords poor-quality monolayers of $\mathbf{1}$.

\section{-Insert Figure 4 here-}

In order to get some atomistic insight into the formation of the studied monolayers, all-atomic Molecular Dynamics (MD) simulations were carried out (see Simulation Methods Section). Atomically-flat gold surfaces with different coverage degrees of 1 were considered, both in vacuo and with water as solvent. All MD simulations were performed at $25^{\circ} \mathrm{C}$ and, wherever present in the simulation, the solvent was kept at 1 atm of pressure. The spatial organization of compound 1 with regard to the surface was characterized by measuring the tilt angle $\alpha$, the dependence of which with molecular coverage is shown in Figure 5a. At very low values (< $\left.1 \mathrm{molec} / \mathrm{nm}^{2}\right)$, molecules of 1 tend to lie roughly flat on the surface $\left(\alpha \approx 0^{\circ}\right)$, with catechol groups adsorbed at the interface, as would be expected for a monolayer in its first formation stages (Figure 5b). Higher surface coverages of $\mathbf{1}$ generate equilibrium configurations with raising tilt angles, so that at about $2 \mathrm{molec} / \mathrm{nm}^{2}$, catechol groups appear substantially desorbed from the gold surface with $\alpha \approx 30^{\circ}$. This trend continues at least up to a surface coverage of ca. 3.6 molecules $/ \mathrm{nm}^{2}$-the largest coverage simulated-, for which a tilt value of $\alpha \approx 60^{\circ}$ is obtained. As can be seen, the effect of the solvent is not very important except at large coverage values, when it tends to induce larger tilts as compared to the in vacuo case.

During the simulations, we have also computed the energy per molecule -the sum of the interactions with other molecules, solvent and surface, plus the conformational energy, and the kinetic energy due to thermal agitation- for each surface coverage value, and hence for each estimated tilt angle. For SAMs in presence of water (Figure 5a), and very low coverages $\left(<1 \mathrm{molec} / \mathrm{nm}^{2}\right)$ the molecular energy is found to be roughly constant and consistent with a 
sparse coverage of independent, randomly oriented and flat-lying molecules (Figure 5b). For higher surface coverages, more favourable intermolecular interactions, bring about a consistent decrease in the molecular energy, concomitant to increasing tilt angles (Figure 5c), suggesting that the spontaneous formation of monolayers of $\mathbf{1}$ should be energetically favoured. According to the MD calculations, the energy seems to reach a minimum at a coverage of about 3.33 molecules $/ \mathrm{nm}^{2}$ (corresponding to a tilt angle of $\alpha \approx 60^{\circ}$ ), meaning this would be the energetically preferred coverage for SAMs made of compound $\mathbf{1}$. The energy per molecule increases for larger coverages, which is attributable to packing constraints and steric interactions between adjacent molecules.

\section{-Insert Figure 5 here-}

\section{Summary}

The spectroscopic characterization (PM-IRRAS, XPS, ellipsometry) of gold substrates coated with catechol-thiol 1 showed the tendency of this molecule to self-assemble on gold surfaces forming monolayers with outward-facing catechol groups. For this, sufficiently prolonged immersion times (i.e. longer than for long-chain alkanethiols) of atomically flat gold surfaces in solutions of 1 were mandatory in order to obtain good quality monolayers. The spontaneous formation of monolayers of $\mathbf{1}$ on gold was supported by theoretical calculations showing that this process should be energetically favoured.

With regard to the average adhesion force of catechol-terminated monolayers at the molecular level, it was found to be five times higher than that of bare gold, and eight times higher than that of an ODT monolayer. Comparison with PDA coated substrates also support the existence of enhanced adhesion for the monolayers, as F-d curves measured on PDA coatings not only showed overall lower adhesion than those of catechol 1 SAMs, but much lower 
reproducibility as well, hinting at their inherent lack of structural and chemical homogeneity. Finally, surface roughness was also shown to impair the final adhesion properties of the monolayer: an increase on the surface roughness led to a severely diminished adhesion force at the molecular scale, although coated 'rough' substrates still showed the ability to organize magnetic nanoparticles on its surface.

\section{Experimental and Simulation Methods}

\section{Synthesis. General Procedures.}

Commercially available reagents were used as received. The solvents were dried by distillation over the appropriate drying agents. All reactions were performed avoiding moisture by standard procedures and under nitrogen atmosphere and monitored by analytical thin-layer chromatography (TLC) using silica gel $60 \mathrm{~F}_{254}$ pre-coated aluminum plates $(0.25$ mm thickness). Flash column chromatography was performed using silica gel $60 \AA$, particle size $35-70 \mu \mathrm{m}$ (230-400 mesh). ${ }^{1} \mathrm{H}-\mathrm{NMR}$ spectra were recorded on Bruker DPX250 (250 MHz), DPX360 (360 MHz) and ARX400 (400 MHz) spectrometers. Proton chemical shifts are reported in ppm $(\delta)\left(\mathrm{CDCl}_{3}, \delta 7.26\right.$ or $\left.\mathrm{CD}_{3} \mathrm{OD}, \delta 3.31\right) .{ }^{13} \mathrm{C}-\mathrm{NMR}$ spectra were recorded on Bruker DPX250 (62.5 MHz) and Bruker DPX360 (90 MHz) spectrometers with complete proton decoupling. Carbon chemical shifts are reported in ppm $(\delta)\left(\mathrm{CDCl}_{3}, \delta 77.0\right.$ or $\mathrm{CD}_{3} \mathrm{OD}$, $\delta$ 49.0). The ATR-IR spectra were recorded in a Tensor 27 (Bruker) combined with an ATR MKII Golden Gate accessory. The melting points (MP) have been determined using a Reichert kofler block and have not been corrected. Synthetic details are given in the Supporting Information.

\section{Surface Modification. General procedures.}

HPLC grade solvents (ACN and EtOH, Panreac) and Mili-Q water $(18.2 \mathrm{~m} \Omega \cdot \mathrm{cm})$ were used for the SAM formation and substrate cleaning processes. Dopamine hydrochloride, Tris-HCl 
and 1-octadecanethiol (ODT, 98\%) were purchased from Aldrich. All the commercially available reagents were used as received.

\section{Preparation of gold substrates.}

Two types of gold substrates have been used in the present study, namely, polycrystalline and epitaxial gold. Polycrystalline gold substrates were obtained by evaporation of gold onto silicon wafers bearing a native oxide layer $\left(\mathrm{Si} / \mathrm{SiO}_{2}\right.$ using an Electron Beam Evaporator (from AJA International Inc). $\mathrm{Si} / \mathrm{SiO}_{2}$ substrates were prepared by initially cutting silicon wafers into $1 \times 1 \mathrm{~cm}^{2}$ pieces. Then, each $\mathrm{Si} / \mathrm{SiO}_{2}$ substrate was washed in an ultrasonic bath for $15 \mathrm{~min}$ in $\mathrm{ACN}, \mathrm{EtOH}$, and Milli-Q water and dried under a nitrogen stream. In the evaporator, the substrates where first coated with a $\mathrm{Ti}(99.99 \%)$ layer $(\sim 10 \mathrm{~nm})$ to act as a primer and then a $\sim 40 \mathrm{~nm}$ layer of $\mathrm{Au}(99.99 \%)$ was deposited on top. During the whole process, the pressure inside the evaporator was $\sim 10^{-8}$ Torr. Prior to incubation, the polycrystalline gold substrates were cleaned by sonication in ACN, EtOH and Milli-Q water and dried in a nitrogen stream. Epitaxial gold $(300 \mathrm{~nm})$ on mica substrates were purchased from Georg Albert PVD and stored under vacuum. Prior to the SAM formation, the epitaxial gold substrates were cleaned by carefully rinsing with acetone, EtOH and Milli-Q water and dried under a nitrogen stream.

\section{SAM formation.}

All the substrates were cleaned in a $\mathrm{UV} / \mathrm{O}_{3}$ cleaner for $10 \mathrm{~min}$ (Novascan Technologies) and immediately immersed in the corresponding solutions. SAM formation was obtained by a standard procedure, as follows. Clean gold substrates were immersed overnight in $1 \mathrm{mM}$ solutions of the corresponding thiols in EtOH. Then, the substrates were rinsed with copious amounts of EtOH and Milli-Q water and dried by a nitrogen flow. For the force-distance measurements, the modified substrates were allowed to dry overnight; for the rest of the experiments, they were immediately used as prepared.

\section{SAM characterization.}


Polarization modulation infrared reflection-absorption spectra were recorded on a FT-IR spectrometer Vertex 70(Bruker) combined with a PMA50 accessory. The angle of incidence during the acquisition of the spectra was $80^{\circ}$. Two separated spectra were recorded with the photoelastic modulator set at $2900 \mathrm{~cm}^{-1}$ for the $\mathrm{OH}$ and $\mathrm{CH}_{2}$ stretching region and at $1600 \mathrm{~cm}^{-}$ ${ }^{1}$ for $\mathrm{C}=\mathrm{C}$ and $\mathrm{CO}$ stretching and $\mathrm{OH}$ bending region.

AFM imaging and adhesion force measurements were performed on an Agilent 5500 AFM/SPM microscope combined with PicoScan software from Agilent Technologies. Contact mode and intermittent contact mode AFM were used for imaging. Intermittent contact mode AFM was performed using beam shaped aluminium-coated silicon cantilevers (Nanosensors) with nominal force constants of $42 \mathrm{~N} \cdot \mathrm{m}^{-1}$ and $7 \mathrm{~nm}$ tip radius. Whereas contact mode AFM and force-distance measurements were carried out using aluminum-coated silicon cantilevers with nominal force constant of $0.2 \mathrm{~N} \cdot \mathrm{m}^{-1}$ and $7 \mathrm{~nm}$ tip radius. After the adhesion experiments, all the cantilevers were calibrated (in sensitivity and spring constant). At least 45 experiments were performed on each sample to provide an average value. Initially, a unique AFM tip was used in order to obtain directly comparable data for the three studied samples. However, we detected that contamination of the tip with the experiments resulted in an increase of the dispersion, and decided to use a new tip for each sample.

X-ray photoelectron spectroscopy was carried out in a Phoibos 150 analyzer (SPECS GmbH, Berlin,Germany)) in ultra-high vacuum conditions (base pressure $1 \cdot 10^{-10} \mathrm{mbar}$ ). A monochromatic $\mathrm{K} \alpha \mathrm{X}$-ray source $(1486.6 \mathrm{eV})$ was used. The spectra were based on photoelectrons with a takeoff angle of $0^{\circ}$ for the S $2 p$ core level and $30^{\circ}$ for the $\mathrm{C} 1 \mathrm{~s}$ core level (takeoff angle considered with respect to the surface normal).

Sprectroscopic Ellipsometry was carried out in a Semilab Sopra GES5E spectroscopic ellipsometer in order to determine the thickness and refractive index of the layered structures. During the investigation the incident angle of the light beam was set to 65,70 or 75 degrees and the wavelength was varied from 300 to $800 \mathrm{~nm}$. Measurements were carried out at least 
three macroscopically spaced points on the sample. Ellipsometric data was fitted with multilayer models using the Semilab's WinElli II analysis software. Clean gold substrates were modelled first. Next, a Cauchy model $(\mathrm{A}=1.49)$ was used to model the organic film.

\section{Polydopamine synthesis and surface coating.}

Polydopamine was obtained following the previously described procedure. ${ }^{[1]}$ Dopamine hydrochloride $(2 \mathrm{mg} / \mathrm{mL})$ was dissolved in $10 \mathrm{mM}$ Tris $\cdot \mathrm{HCl}(\mathrm{pH} 8.6)$ solution. The substrates were placed in vertical orientation into the mixture solution and for 1 hour while stirring to minimize non-specific deposition. Afterwards, the substrates were rinsed with Milli-Q water and dried under a nitrogen stream.

\section{Iron oxide nanoparticles: synthesis and deposition.}

Maghemite nanoparticles were obtained by coprecipitation. $\mathrm{FeCl}_{2} \cdot 4 \mathrm{H}_{2} \mathrm{O}(10.21 \mathrm{~g}, 52 \mathrm{mmol})$ was dissolved in Milli-Q water (1L). Then, a solution of $\mathrm{FeCl}_{3} \cdot 6 \mathrm{H}_{2} \mathrm{O}(28.35 \mathrm{~g}, 104 \mathrm{mmol})$ in $\mathrm{HCl} 1.5 \mathrm{M}(57 \mathrm{~mL})$ was added under strong stirring to yield nanometric magnetite $\left(\mathrm{Fe}_{3} \mathrm{O}_{4}\right)$. To the previous solution, $25 \% \mathrm{NH}_{3}(100 \mathrm{~mL})$ was added and stirring was stopped after $15 \mathrm{~min}$, followed by a two days decantation on a magnet. The black flocculate was dispersed to a $2 \mathrm{M}$ $\mathrm{HNO}_{3}$ solution and stirred for 2-3 min. After decantation, the particles were oxidized to maghemite by adding a solution of $\mathrm{Fe}\left(\mathrm{NO}_{3}\right)_{3} \cdot 9 \mathrm{H}_{2} \mathrm{O}(27.2 \mathrm{~g}, 67.3 \mathrm{mmol})$ in Milli-Q water (200 $\mathrm{mL}$ ) and stirring for $30 \mathrm{~min}$ at $100^{\circ} \mathrm{C}$. After that, a magnetic decantation (2-3h) was carried out and the product was dispersed to a $2 \mathrm{M} \mathrm{HNO}_{3}$ solution and stirred during $15 \mathrm{~min}$. The average diameter of the obtained magnetic nanoparticles was 8-10 nm, as estimated form transmission electron microscopy (TEM, Hitachi H-7000) images. The obtained nanoparticle suspension was diluted to a concentration of $\mathrm{Fe}_{2} \mathrm{O}_{3} \sim 65 \mathrm{mg} / \mathrm{L}$ in Milli-Q water and it was sonicated for $15 \mathrm{~min}$. Then, the gold substrates were immersed in the suspension, sonicated during $15 \mathrm{~min}$ and rinsed with Milli-Q water and dried by nitrogen gas. To observe the results of these experiments, scanning electron microscopy (Magellan 400L, Quanta 650 FEG, FEI; MERLIN®, Zeiss) was used. 


\section{Dip-Pen Nanolithography.}

DPN experiments were performed with a commercial dip-pen writer; NSCRIPTOR ${ }^{\mathrm{TM}}$ DPN® System (Nanoink Inc.). Commercial silicon nitride Type A tips (Nanoink Inc.) with a spring constant of $0.05 \mathrm{~N} \cdot \mathrm{m}^{-1}$ were used for patterning. All DPN experiments were carried out under ambient conditions ( $40 \%$ relative humidity, room temperature). For the localized monolayer formation, thiol-coated tips were obtained following a previously described method form Nanoink Inc. Briefly, a UV/O 3 cleaned silicon nitride tip (Type A) was immersed in a saturated solution of 4-(6'-mercaptohexyl)catechol (1) in ACN for about 10 seconds and dried with gentle nitrogen flow. After that, the tip was exposed to water vapor for 5 min and then left to dry. Once dry, the tip was re-dipped again in the same solution and blow-dried with nitrogen. It is worth noting that epitaxial gold surfaces are quite fragile and soft, so careful adjust of the parameters of the processes, such as laser alignment and deflection setpoint, was required when performing the lithographies and subsequent characterization steps to avoid indentation of the surface.

\section{Molecular Dynamics Simulations.}

Molecular Dynamics (MD) simulations are based on the numerical solution of the Newtonian equations of motion for all atoms of a molecular system constrained to the given thermodynamic conditions. All MD simulations were performed using the NAMD software, ${ }^{[33]}$ version 2.9 running in parallel at the Finisterrae Supercomputer (CESGA Supercomputing Center). The equations of motion were solved with a 2 fs time step. The temperature was kept constant at $25{ }^{\circ} \mathrm{C}$ using the Langevin thermostat with a relaxation constant of $1 \mathrm{ps}^{-1}$. In simulations with water as a solvent, the liquid was also maintained at constant pressure of 1 atm employing the Nosé-Hoover-Langevin piston in the vertical direction with an oscillation period of $100 \mathrm{fs}$ and a decay time of $50 \mathrm{fs}$ (which are standard parameters for NAMD). Periodic boundary conditions in all directions were employed in all our simulations. The snapshots of the simulations and the energy calculations were obtained from the MD 
trajectories by using the Visual Molecular Dynamics (VMD) software. ${ }^{34}$ More details are given in the Supplementary Material.

\section{Acknowledgments}

This work was supported by MICINN through projects MAT2012-38318-C03-02, MAT201238319-C02-01 and CONSOLIDER NANOSELECT CSD 2007-00041. We also acknowledge the CESGA Supercomputing Center for computational time at the Finisterrae Supercomputer and technical assistance and the Common Services and Equipment team for their help with the PM-IRRAS and XPS experiments. M. G. thanks the CSIC for a predoctoral grant. 

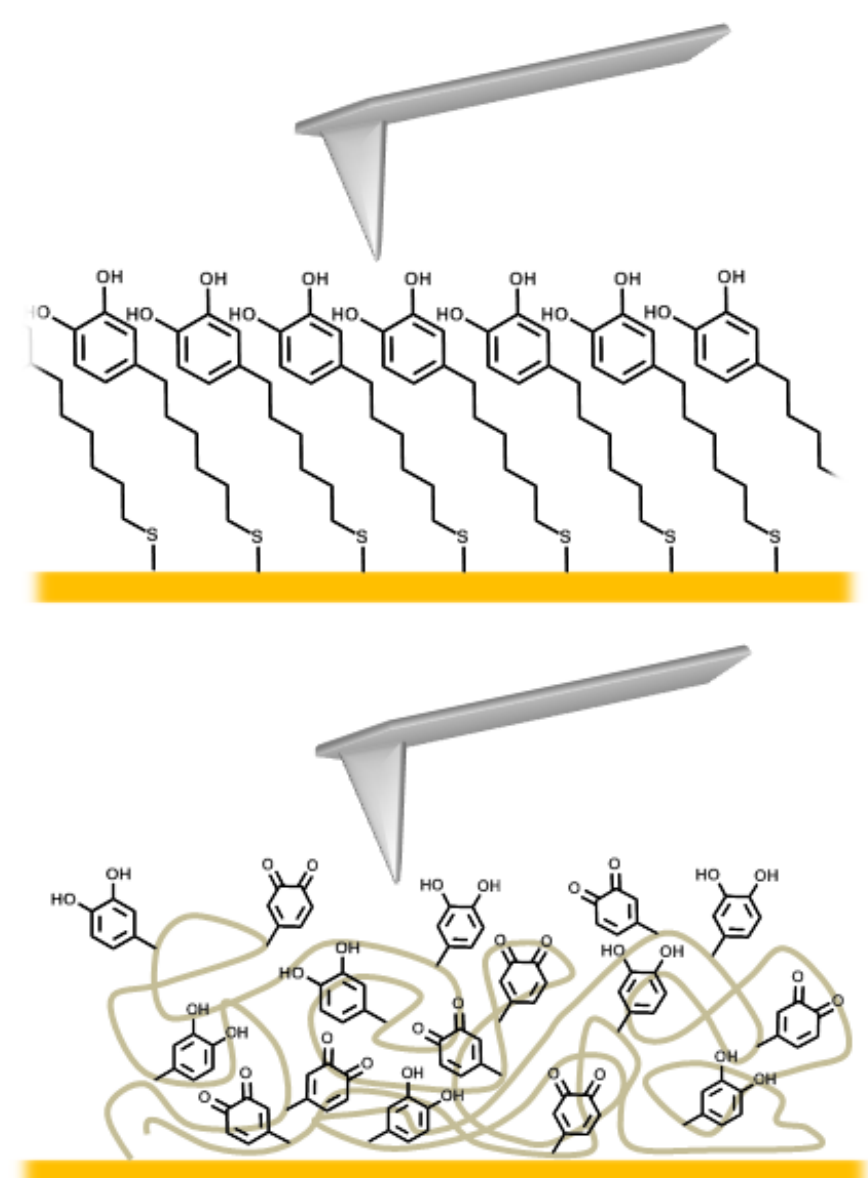

Scheme 1. Schematic representation of the studied surfaces. a) SAM of compund 1 on gold b) Polydopamine coating showing the presence of catechol and quinone moieties in a disordered manner. 

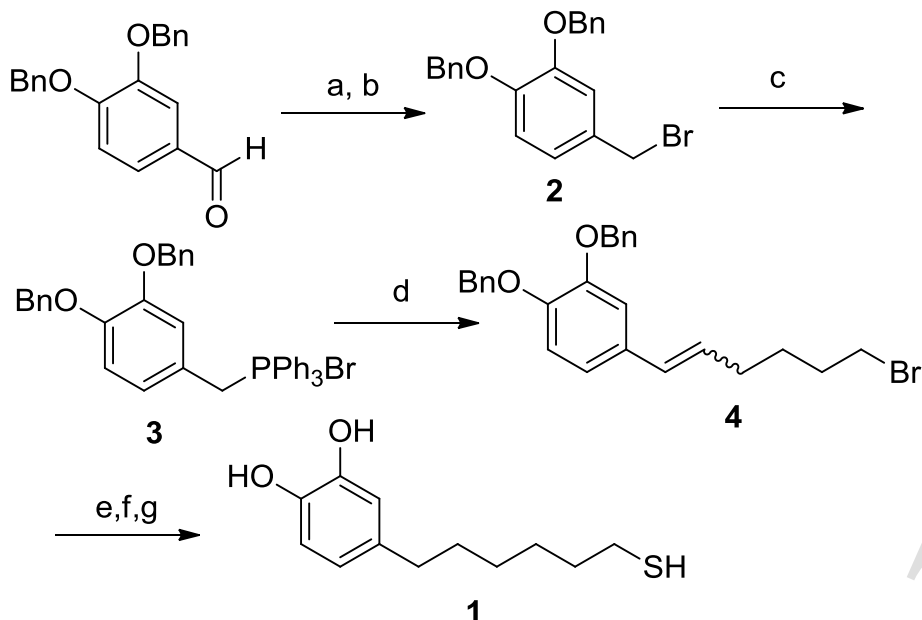

Scheme 2. Synthesis of compound 1: (a) $\mathrm{NaBH}_{4}, \mathrm{MeOH}, \mathrm{CH}_{2} \mathrm{Cl}_{2}, \mathrm{rt}, 4$ h, 97\%; (b) $\mathrm{PBr}_{3}$, $\mathrm{CH}_{2} \mathrm{Cl}_{2}$, rt, $3 \mathrm{~h}, 90 \%$; (c) $\mathrm{PPh}_{3}, \mathrm{CH}_{2} \mathrm{Cl}_{2}$, reflux, 4 h, 97\%; (d) 5-bromopentanal, $\mathrm{K}_{2} \mathrm{CO}_{3}$, $\mathrm{CH}_{2} \mathrm{Cl}_{2}$, rt, 2 h, 50\%; e) $\mathrm{H}_{2}, \mathrm{Pd} / \mathrm{C}$, EtAcO, HAcO, rt, 24 h, 86\%; f) KSAc, DMF, rt, 6 h, 85\%; g) $0.2 \mathrm{M} \mathrm{NaOH}, \mathrm{EtOH}, \mathrm{rt}, 3 \mathrm{~h}, 49 \%$. 

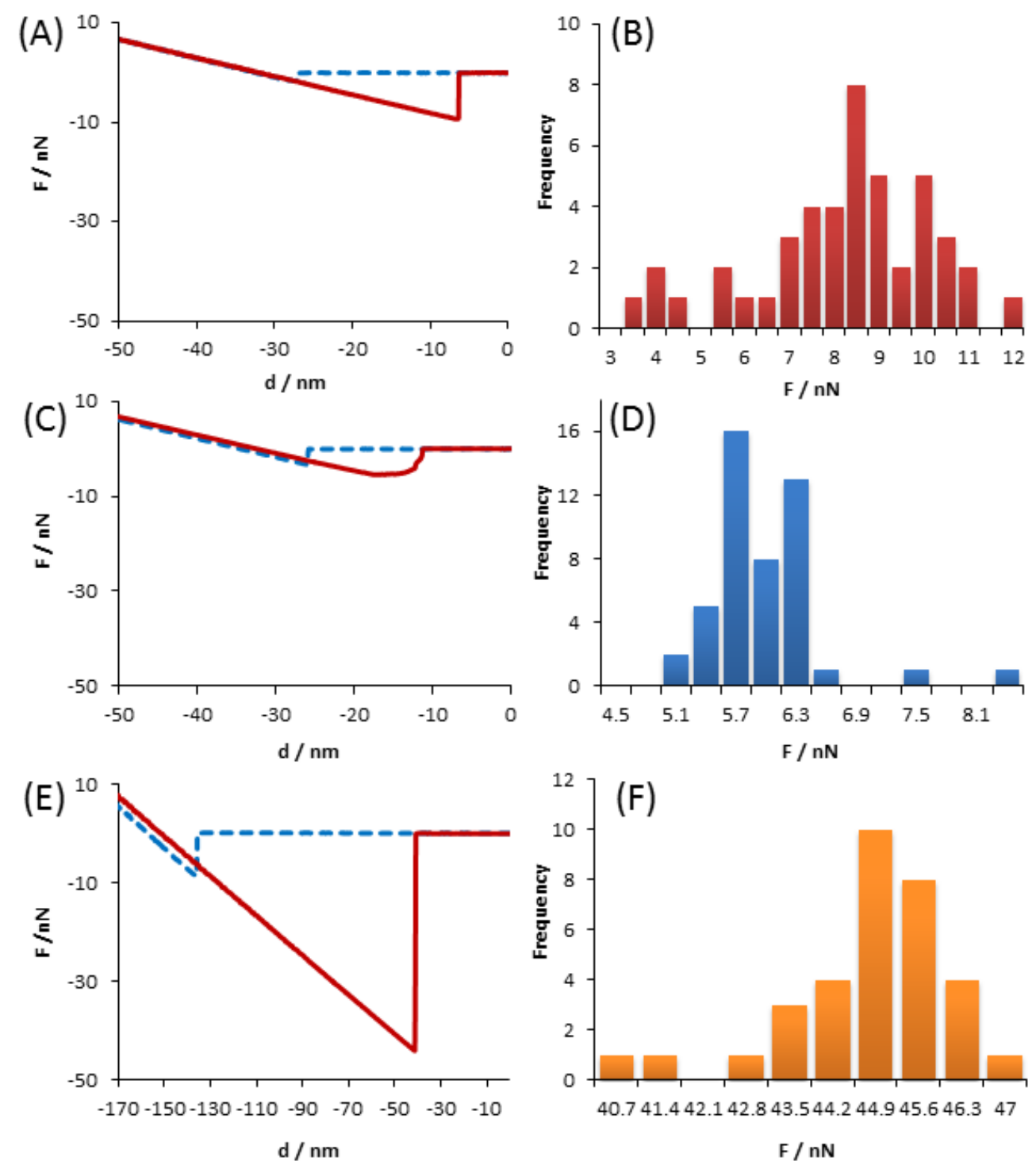

Figure 1. Adhesion measurements on epitaxial gold on mica substrates. Representative force curves for each of the substrates are displayed in the left column. The blue dashed line and the red solid line indicate the approach and retract signals, respectively. Histograms of the adhesion force values are showed in the right column. (A) and (B) unmodified gold, (C) and (D) ODT monolayer, (E) and (F) 1 monolayer. 

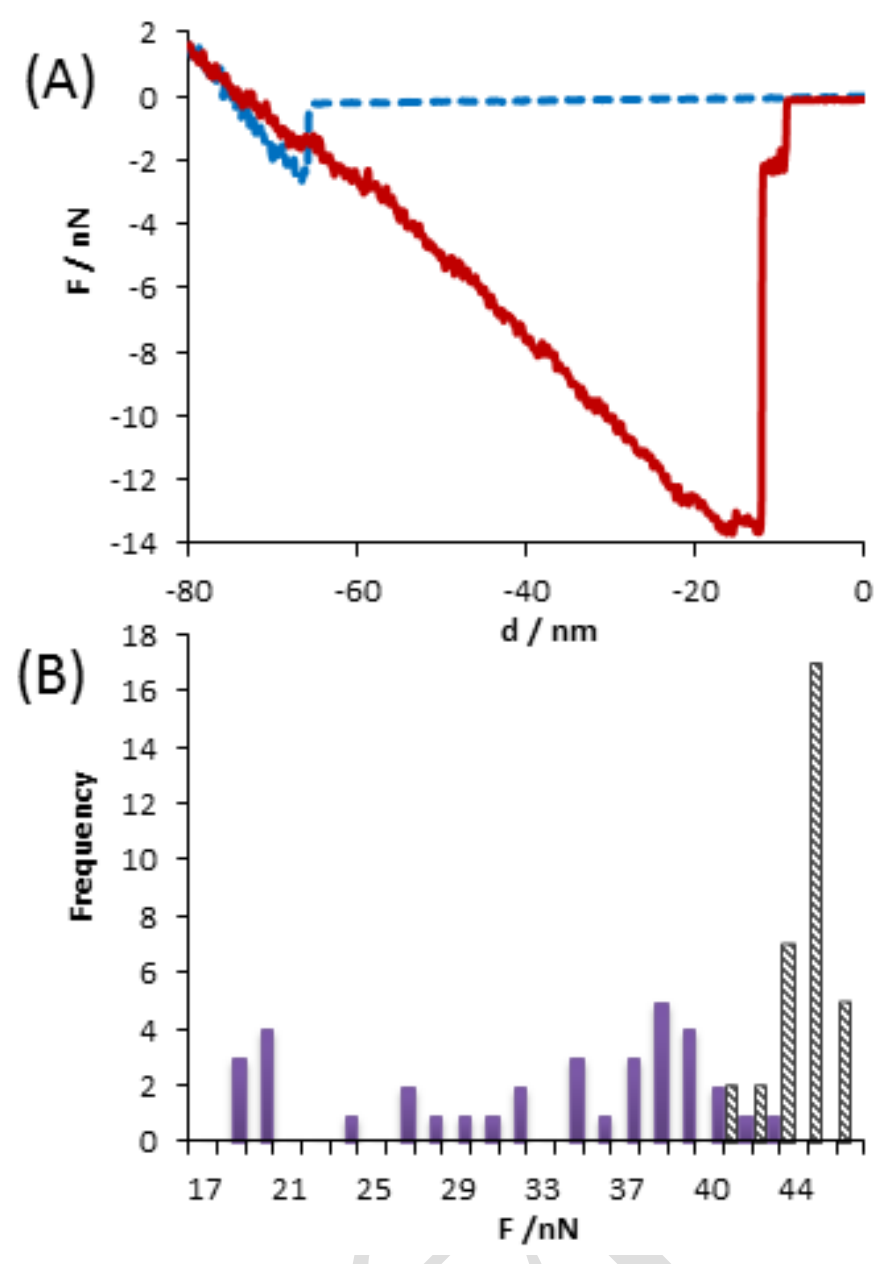

Figure 2. Adhesion measurements on PDOPA coatings in contrast with X1 SAM. (A) Adhesion curve recorded on a PDOPA coating showing multiple jump-out. The blue dashed line indicates the approach signal and the red solid line shows the retract signal. (B) Histogram of the adhesion measurements on PDOPA (solid purple bars) and X1 SAM (striped grey bars). The monolayer shows less dispersion in the results and higher average adhesion force due to its homogeneity and high density of adhesive moieties. 

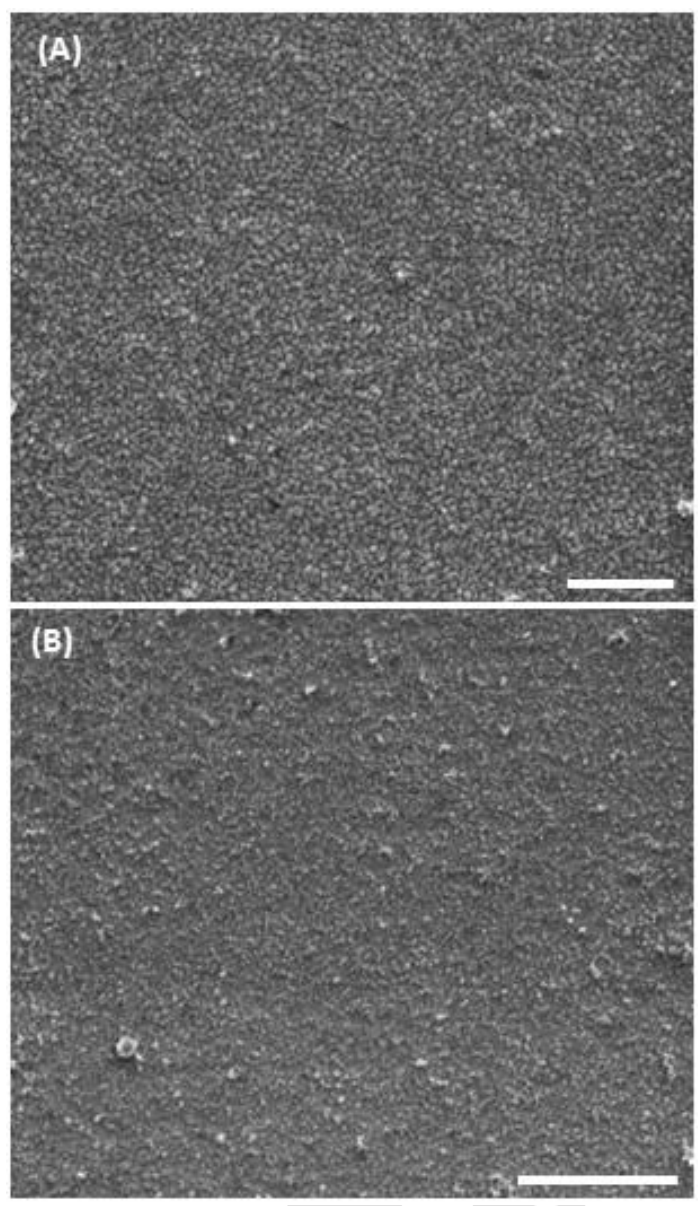

Figure 3. Adsorption of magnetic nanoparticles on functionalised substrates. SEM images of (A) bare polycrystalline $\mathrm{Au}$ on $\mathrm{Si}$ substrate where the gold grains can be clearly seen and barely any magnetic nanoparticles are observed on the surface, and (B) 1-modified polycrystalline gold substrate. In this case, gold grains are not visible, indicating that the surface is fully covered by nanoparticles. The images were obtained after sonication of the substrates in a dispersion of nanoparticles for 15 min followed by rinsing with $\mathrm{H}_{2} \mathrm{O}$. Scale bars are $1 \mu \mathrm{m}$. 


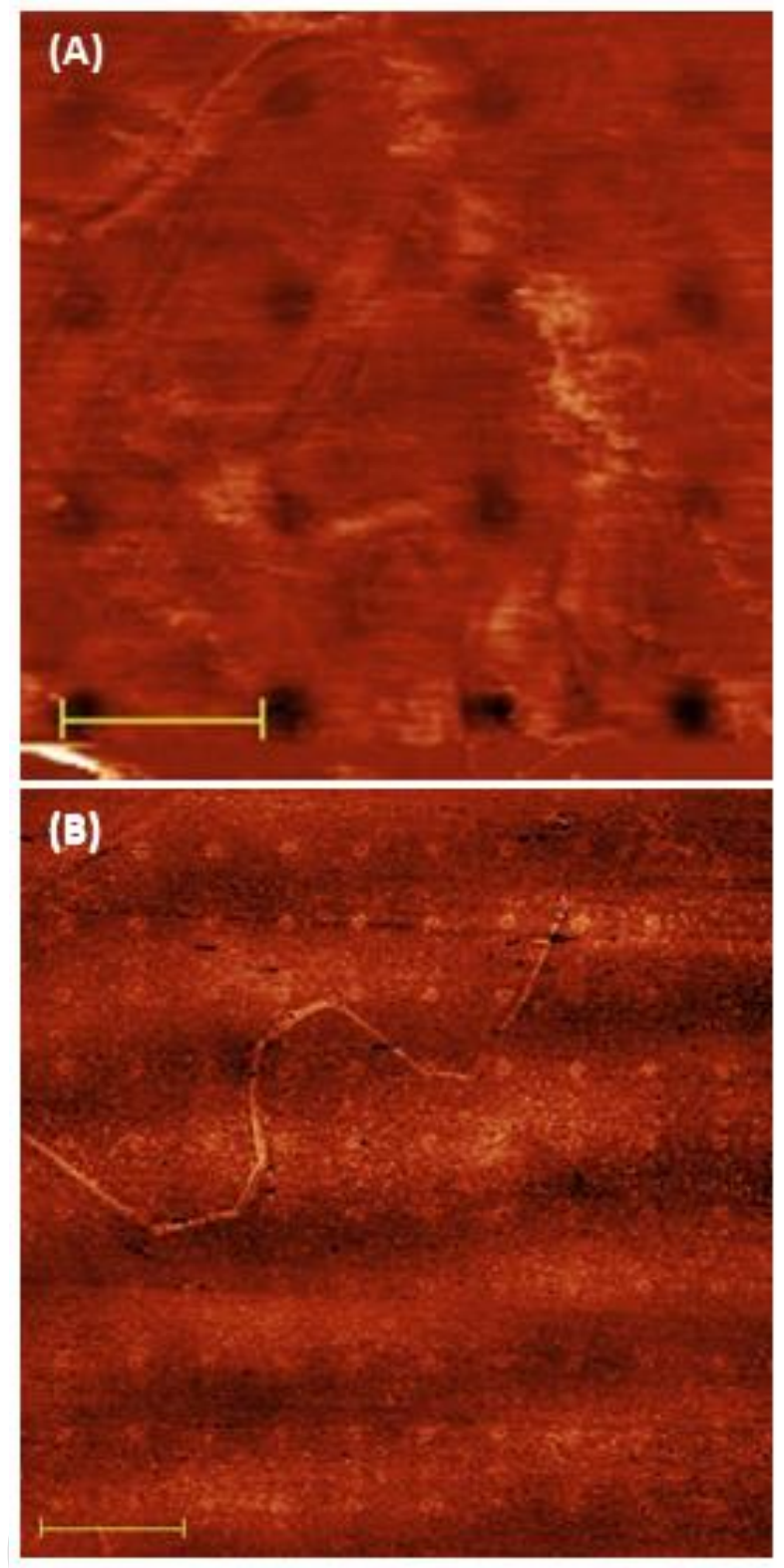

Figure 4. Dot arrays of compound 1 on epitaxial gold on mica. (A) LFM image of a freshly deposited array of compound 1 obtained with the same coated tip used for the lithography. (B) LFM image obtained by contact-mode AFM. Scale bars are $2 \mu \mathrm{m}$. 
(A)

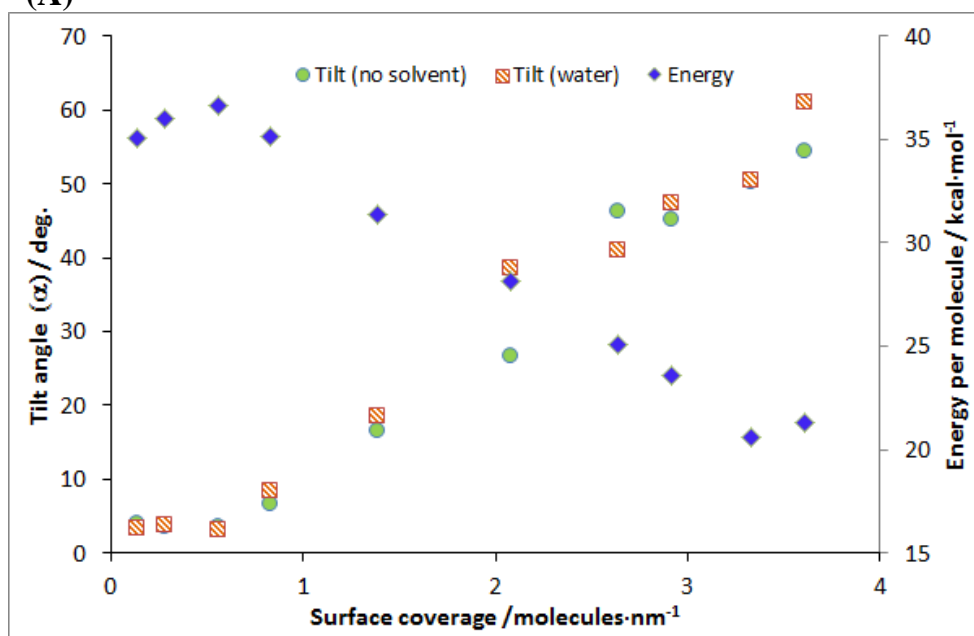

(B)

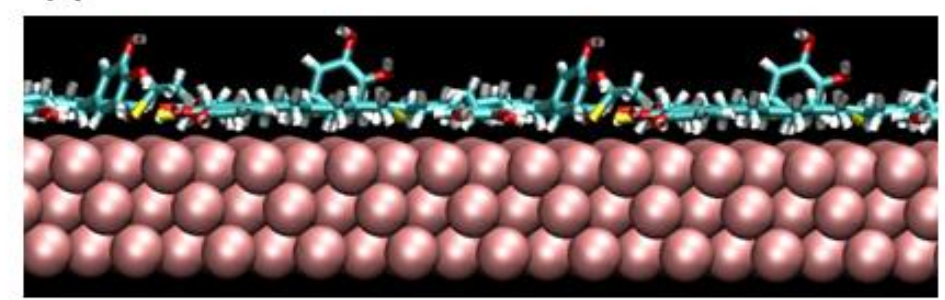

(C)

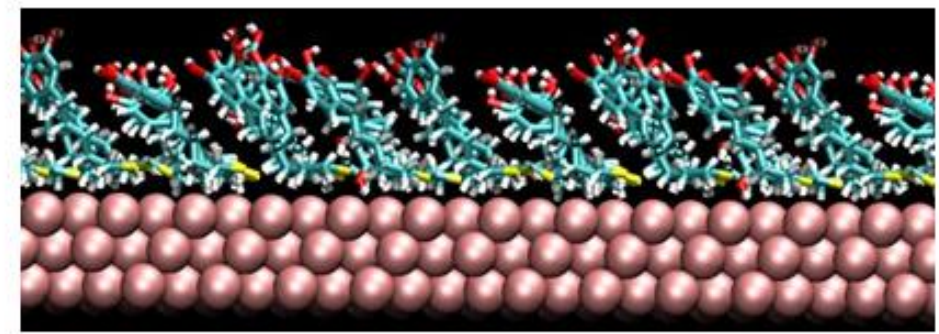

Figure 5. Results from MD simulations. The angle between the catechol group of adsorbed compound 1 molecules and the gold surface (left axis), and the energy per molecule calculated vs surface coverage (blue diamonds, right axis), are shown. Stripped orange squares correspond to simulations in water and filled green circles correspond to simulations without solvent. The snapshots correspond to simulations with a surface coverage of 0.83 molecules $/ \mathrm{nm}^{2}$ (B) and 2.64 molecules $/ \mathrm{nm}^{2}$ (C) with water as solvent (water molecules are not shown for clarity). 


\section{Reference}

[1] H. Lee, S. M. Dellatore, W. M. Miller, P. B. Messersmith, Science 2007, 318, 426.

[2] S. Kang, J. Rho, I. S. Choi, P. B. Messersmith, H. Lee, J. Am. Chem. Soc. 2009, 131, 13224

[3] a) C. Tao, S. Yang, J. Zhang, J. Wang, Appl. Surf. Sci. 2009, 256, 294; b) H.Y. Hu, B. Yu, Q. Ye, Y.S. Gu, F. Zhou, Carbon 2010, 48, 2347; c) S. Hong, K.Y. Kim, H. J. Wook, S.Y. Park, K. D. Lee, D.Y. Lee, H. Lee, Nanomedicine 2011, 6, 793; d) S. H. Ku, C. B. Park, Biomaterials 2010, 31, 9431; e) Y.M. Shin, H. Park, H. Shin, Macromol. Res. 2011, 19, 835; f) S. H. Yang, S. M. Kang, K. B. Lee, T. D. Chung, H. Lee, I. S. Choi, J. Am. Chem. Soc. 2011, 133, 2795.

[4] J. H. Waite, Nat. Mater.2008, 7, 8.

[5]Y. B. Lee, Y. M. Shin, J. Lee, J. K. Kang, J. Park, H. Shin, Biomaterials 2012, 33, 8343.

[6] H. Chien, W. Kuo, M. Wang, S. Tsai, W. Tsai, Langmuir 2012, 28, 5775.

[7]J. Feng, P. Zhang, A. Wang, Q. Liao, J. Xi, J. Chen, New J. Chem. 2012, 36, 148.

[8] J. Cui, Y. Yan, G. K. Such, K. Liang, C. J. Ochs, A. Postma, F. Caruso, Biomacromolecules 2012, 13, 2228.

[9] J. T. Arena, B. McCloskey, B. D. Freeman, J. R. McCutcheon, J. Membrane Sci. 2011, $375,55$.

[10] J. Saiz-Poseu , J. Sedó , B. García, C. Benaiges, T. Parella, R.Alibés, J. Hernando, F. Busqué,D.Ruiz-Molina, Adv. Mater. 2013, 25, 2066-2070

[11] a) J. Sedó, J. Saiz-Poseu, F. Busqué, D. Ruiz-Molina, Adv. Mater. 2013, 25, 653. b) F. Bernsmann, A. Ponche, C. Ringwald, J. Hemmerle, J. Raya, B. Bechinger, J. Voegel, P. Schaaf, V. Ball, J. Phys. Chem. C 2009, 113, 8234. C) D. R. Dreyer, D. J. Miller, B. D. Freeman, D. R. Paul, C. W. Bielawski, Langmuir 2012, 28, 6428 
[12] D. L. Allara, in Polymer Surfaces and Interfaces, Vol. 2 (Eds: W. J. Feast, H. S. Munro, R. W., Richards), Wiley, Chichester, Great Britain 1993, p 27.

[13] J. C. Love, L. A. Estroff, J. K. Kriebel, R. G. Nuzzo, G. M. Whitesides, Chem. Rev. 2005, 105, 1103.

[14] a) D. Hojo, T. Togashi, D. Iwasa, T. Arita, K. Minami, S, Takami, T. Adschiri, Chem. Mater. 2010, 22, 1862. b) B. Basnar, J. Xu, I. Willner Langmuir 2007, 23, 2293.

[15] a) A. Salmanipour, M. A. Taher, Analyst 2011, 136, 545. b) J. Kang, L. Zhuo, X. Lu, X. Wang, J. Solid State Electr.2005, 9, 114. c) K. Nakano, K. Ohkubo, H. Taira, M, Takagi, T. Imato, Anal. Chim. Acta 2008, 619, 30. d) N. J. Simmons, K. O. A. Chin, J. A. Harnisch, B. Vaidya, W. S. Trahanovsky, M. D. Porter, R. J. Angelici, J. Electroanal. Chem. 2000, 482, 178.

[16] K. A. Brun, A. Linden, H. Heimgartmer, Helv. Chim.Acta .2002, 85, 3422.

[17] M. Miesch, L. Miesch, P. Horvatovich, D. Burnouf, H. Delincée, A. Hartwing, F. Raul, D. Werner, E. Marchioni, Rad. Phys. Chem. 2002, 65, 233.

[18] C. Vahlberg, M. Linares, S. Villaume, P. Norman, K. Uvdal, J. Phys. Chem. C 2011, 115, 165.

[19] M. Weinhold, S. Soubatch, R. Temirov, M. Rohlfing, B. Jastorff, F. S. Tautz, C. Doose, J. Phys. Chem. B 2006, 110, 23756.

[20] M. D. Porter, Anal. Chem. 1988, 60, 1143.

[21] J. A. Mielczarski, J. Dong, E. Mielczarski, J. Phys. Chem. B 2008, 112, 5228.

[22] A. Ulman, An Introduction to Ultrathin Organic Films: From Langmuir-Blodgett to SelfAssembly, Academic Press, 1991.

[23 ] H.G. Tompkins H.G., W. A. McGahan W.A., Spectroscopic Ellipsometry and reflectometry: A User's Guide, John Wiley \& Sons, Inc., 1999.

[24] H. Lee, N. F. Scherer, P. B. Messersmith, Proc. Natl. Acad. Sci. USA 2006, 103, 12999. 
[25] B. Cappella, G. Dietler, Surf. Sci. Rep.1999, 34, 1.

[26] A. Verdaguer, G. M. Sacha, H. Bluhm, M. Salmeron, Chem. Rev. 2006, 106, 1478.

[27]D. Del Frari, J. Bour, V. Ball, V. Toniazzo, D. Ruch, Polym. Degrad. Stabil 2012, 97, 1844.

[28] K. P. Browne, B. A. Grzybowski, Langmuir 2011, 27, 1246.

[29] A. Ulman Chem. Rev. 1996, 96, 1533-1554.

[30] R. D. Piner, J. Zhun, F. Xu, S. Hong, C. A. Mirkin, Science 1999, 283, 661.

[31] a) R. J. Barsotti, Jr., M. S. O’Connell, F. Stellacci, Langmuir 2004, 20, 4795. b) P.

Campiglio, M. Camplone, A. Sassella, J. Phys. Chem. C 2009, 113, 8329.

[32] C. Xu, R. L. Jones, J. D. Batteas, Scanning, 2008, 30, 106.

[33] J. C. Phillips, R. Braun, W. Wang, J. Gumbart, E. Tajkhorshid, E. Villa, C. Chipot, R. D. Skeel, L. Kale and K. Schulten, J. Comput. Chem., 2005, 26, 1781.

[34] W. Humphrey, A. Dalke, K. J. Schulten, Molec. Graphics, 1996, 14, 33. 